\title{
A Large Spontaneous Abdominal Bruise in a 60-Year-Old Woman: A Case Report
}

\author{
Sujatha Mogilia Timothy Woodlock ${ }^{a, b}$ \\ ${ }^{a}$ Department of Medicine, Unity Health System, and ${ }^{b}$ Department of Medicine, University of Rochester School of \\ Medicine and Dentistry, Rochester, N.Y., USA
}

\section{Key Words}

Acquired hemophilia A - Coagulation disorder - Factor VIII • Spontaneous bruising

\begin{abstract}
Objective: To report a case of severe abdominal bruising successfully diagnosed using systematic hemostatic investigations. Clinical Presentation and Intervention: A 60-yearold woman developed a large spontaneous nontraumatic, painless bruise over the right lower abdominal wall. Computed tomographic scan of the abdomen showed a subcutaneous hematoma beneath the site of the obvious bruise diagnosed as a hematoma. Laboratory tests revealed an activated partial thromboplastin time of $53 \mathrm{~s}$ and a factor VIII level of $<1 \%$, consistent with the diagnosis of acquired hemophilia A. The patient was treated with steroids and immunosuppressants and her factor VIII levels gradually normalized. Conclusion: This case report showed that whenever there is spontaneous bruising, factor VIII deficiency should be considered as one of the important differential diagnosis.
\end{abstract}

Copyright ๑ 2011 S. Karger AG, Basel

\section{Introduction}

The evaluation of unexpected large or potentially lifethreatening bruises requires an assessment of vascular integrity, platelet functions and plasma factors $[1,2]$. Pa- tients with platelet function abnormalities frequently have mucous membrane bleeding, bleeding immediately after injury, superficial ecchymoses and petechiae [2]. Patients with coagulation abnormalities may present with deep soft tissue bleeding, or with deep hematomas which clinically may have a delayed appearance and are often more severe, with large ecchymoses. Platelet disorders and coagulation abnormalities should always be considered when the bruise is located in areas which are difficult to traumatize [1]. A detailed history should be obtained and a careful physical examination, complete blood count and peripheral blood smear should be performed in all patients before further investigations are carried out [2]. Unless the patient has a significant history of the suggestive problem, the estimation of platelet count, bleeding time, prothrombin time, partial thromboplastin time, and factor XIII assay constitutes a list of the screening tests [2]. We describe a case of severe abdominal bruising successfully diagnosed and treated using systematic hemostatic investigations.

\section{Case Report}

A 60-year-old woman developed a large spontaneous, nontraumatic, painless bruise over the right lower abdominal wall. The lesion started as a small knot and progressively increased in size (fig. 1). Past medical history revealed fibromyalgia, spinal stenosis, bilateral hip bursitis, and hypercholesterolemia. Surgical history included tonsillectomy, temporomandibular joint sur-

\section{KARGER \\ Fax +4161306 1234 \\ E-Mail karger@karger.ch}

www.karger.com
(C) 2011 S. Karger AG, Basel

$1011-7571 / 12 / 0211-0086 \$ 38.00 / 0$

Accessible online at:

www.karger.com/mpp
Sujatha Mogili, MD

Unity Health System, 1555 Long Pond Rd

Rochester, NY 14626 (USA)

Tel. +1 5852816874

E-Mailsmogili@unityhealth.org 


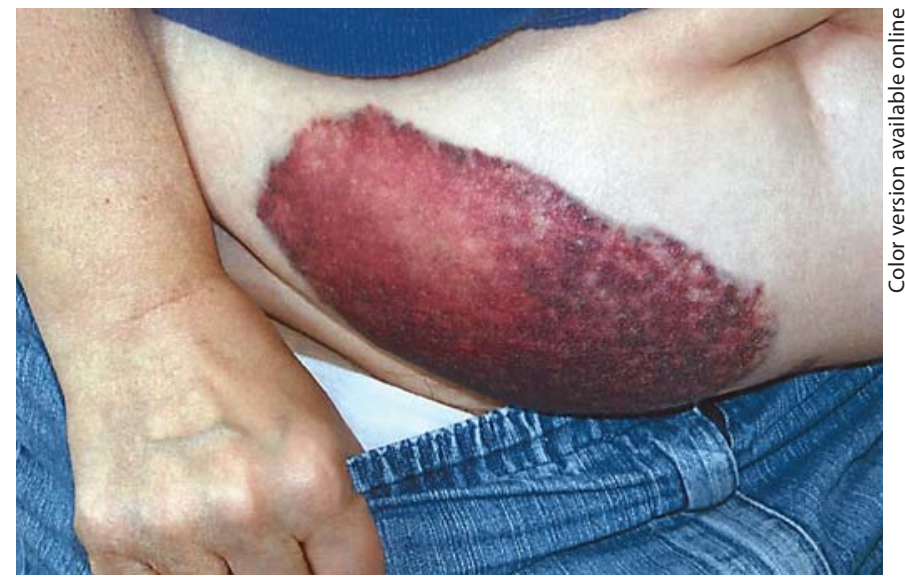

Fig. 1. Spontaneous bruising in the anterior abdominal wall.

gery, cholecystectomy, and breast biopsy for a benign mass. She is a housewife, mother of 2 children and there was no family history of abnormal bleeding. Also there was no history of abnormal bleeding with previous surgeries or during deliveries. Medications were omeprazole, amitriptyline, and lovastatin. She did not smoke, nor did she use alcohol or drugs. Other pertinent positive clinical symptoms were myalgia and dry mouth. On physical examination, she was afebrile with a blood pressure of 140/90 mm $\mathrm{Hg}$, a pulse rate of $86 / \mathrm{min}$, and a dry oral mucosa. Cardiac examination revealed normal heart sounds without a murmur or rubs. The lung auscultation was unremarkable. Abdominal examination revealed $7 \times 6 \mathrm{~cm}$ ecchymosis over the right anterior abdominal wall. The abdomen was soft with mild tenderness over the bruise mark. There were no palpable masses or organomegaly. The extremities had no edema or calf tenderness. No other bruise marks or joint swellings were noted.

The laboratory results were as follows: white blood count, $5.1 \times 10^{3} / \mathrm{mm}^{3}$; hemoglobin, $11.6 \mathrm{~g} / \mathrm{dl}$; platelet count, $238 \times 10^{3} /$ $\mathrm{mm}^{3}$. Liver and renal functions were normal. Her prothrombin time was $11.3 \mathrm{~s}$ with an international normalized ratio of 1.1. The antinuclear antibody titer was 1:40 with a diffuse pattern. Antiextractable nuclear antigen, anticardiolipin, C-reactive protein, C3, C4, total complement, peripheral antineutrophil cytoplasmic antibodies, cytoplasmic antineutrophil cytoplasmic antibodies, Sjögren's syndrome antibodies (anti-SSA, anti-SSB), angiotensinconverting enzyme and rheumatoid factor were not consistent with any defined autoimmune illness. The activated partial thromboplastin time (APTT) was $53 \mathrm{~s}$ (normal <35), and computed tomography scan of the abdomen and pelvis showed a subcutaneous hematoma measuring $2.8 \times 3.1 \mathrm{~cm}$ beneath the site of the obvious bruise. The differential diagnosis includes deficiency or inhibitors of factors VIII, IX and XI or antiphospholipid antibody.

Her factor VIII level was $<1 \%$ (normal $50-180 \%$ ), consistent with acquired hemophilia A. The patient was started on treatment with prednisone $40 \mathrm{mg} /$ day and Cytoxan $50 \mathrm{mg} /$ day until the bleeding resolved. The doses of both medications were then gradually reduced. The patient underwent a workup for lymphoma/ cancer with a chest X-ray, computed tomography scan, and bone

A Case Report of a Large Spontaneous

Abdominal Bruise marrow biopsy, all of which were normal. After the treatment, the APTT levels normalized within a few weeks and her factor VIII activity increased from 1 to $>21 \%$ with no additional bleeding episodes. She was followed up weekly, then monthly as bleeding symptoms decreased and factor VIII levels normalized.

\section{Discussion}

Acquired hemophilia $\mathrm{A}$ is a rare disease associated with severe bleeding complications. It is typically caused by a congenital deficiency of factor VIII, but an acquired form due to inhibitors to factor VIII occurs later in life [3]. The term 'acquired hemophilia' is derived from the fact that the inhibitors to factor VIII create a functional coagulation factor VIII deficiency in individuals who have no prior history of bleeding [4]. Unlike congenital hemophilia A, where hemarthrosis is a typical feature, the bleeding pattern in acquired hemophilia $A$ is different in the sense that these patients experience hemorrhages into the skin, muscles or soft tissues, and mucous membranes, as presented in our case. Moreover, there is no known genetic inheritance pattern and thus this condition occurs equally in men and women $[5,6]$. The incidence of acquired hemophilia increases with age (the median age at presentation is somewhere between 60 and 67 years) as in the case of the 60-year-old woman. An underlying pathology like autoimmune disorders, malignancy, or pregnancy is observed in $50 \%$ of cases, but since all these workups were negative, our case falls into the remaining $50 \%$ of cases that are idiopathic [4]. The timely detection of this condition is critical, since early therapy directed towards achieving hemostasis and inhibitor eradication is life-saving [3]. The diagnosis of acquired hemophilia $\mathrm{A}$ in a patient with no previous personal or family history of bleeding is typically based on two factors: prolonged APTT and a normal prothrombin time indicating an abnormality in the early part of the intrinsic coagulation cascade. In mixing studies, correction of APTT implies a deficiency, whereas persistence of an abnormally prolonged APTT suggests the presence of an inhibitor [7]. In our case, the patient's APTT was $53 \mathrm{~s}$ with factor VIII levels of $<1 \%$ indicating acquired hemophilia A. Treatment of acquired hemophilia is aimed at controlling hemorrhage and ameliorating autoantibody formation [8]. Therapy includes immunosuppression with high-dose corticosteroids, and cyclophosphamide, or with factor VIII supplements [8]. The patient in our case was treated with prednisone $40 \mathrm{mg} /$ day and Cytoxan $50 \mathrm{mg} /$ day until the bleeding resolved and her factor VIII 
level normalized. During the acute phase, patients with low-titer inhibitors can be treated using human factor VIII concentrates, whereas in patients with high titer it is not possible to overcome the inhibiting activity by raising the factor VIII level. Therefore, factor VIII bypassing agents, such as activated prothrombin complex concentrates or recombinant activated factor VII, are used [4].

\section{Conclusion}

This report showed a case of acquired factor VIII deficiency presenting as a single large ecchymosis. It also demonstrated that awareness of how hemophilia A presents, and the initiation of a thorough and timely workup saved her life.
References
1 Karnath B: Easy bruising and bleeding in the adult patient: a sign of underlying disease. Hosp Physician 2005;41:35-39.

2 Garvey B: Easy bruising in women. Can Fam Physician 1984;30:1841.

-3 Ma AD, Carrizosa D: Acquired factor VIII inhibitors: pathophysiology and treatment. Hematology Am Soc Hematol Educ Program 2006:432-437.

4 Franchini M, Lippi G: Acquired factor VIII inhibitors. Blood 2008;112:250-255.

5 Boggio LN, Green D: Acquired hemophilia. Rev Clin Exp Hematol 2001;5:389-404.
6 Green D, Lechner K: A survey of 215 nonhemophilic patients with inhibitors to factor VIII. Thromb Haemost 1981;45:200-203.

$\checkmark 7$ Delgado J, Jimenez-Yuste V, Hernandez-Navarro F, Villar A: Acquired hemophilia: review and meta-analysis focused on therapy and prognostic factors. Br J Hematol 2003; 121:21-35.

8 Bruggers CS, Bleak S: Successful treatment of acquired factor VIII deficiency in a child using activated factor VII concentrates: case report and review of the literature. J Pediatr Hematol Oncol 2003;25:578-580. 